PROCEEDINGS OF THE

AMERICAN MATHEMATICAL SOCIETY

Volume 127, Number 9, Pages 2653-2655

S 0002-9939(99)05423-4

Article electronically published on May 19, 1999

\title{
EULER CHARACTERISTIC OF THE MILNOR FIBRE OF PLANE SINGULARITIES
}

\author{
A. MELLE-HERNÁNDEZ
}

(Communicated by Ron Donagi)

\begin{abstract}
We give a formula for the Euler characteristic of the Milnor fibre of any analytic function $f$ of two variables. This formula depends on the intersection multiplicities, the Milnor numbers and the powers of the branches of the germ of the curve defined by $f$. The goal of the formula is that it use neither the resolution nor the deformations of $f$. Moreover, it can be use for giving an algorithm to compute it.
\end{abstract}

\section{INTRODUCTION}

In this note we deal with germs of analytic functions $f$ of two complex variables with $f(0)=0$ and its factorization $f=f_{1}^{q_{1}} \cdots f_{r}^{q_{r}}$ into irreducible factors, such that $f_{i} / f_{j}, 1 \leq i, j \leq r$, are as power series not units. Let $(C, 0)$ be the germ of the plane curve defined by the local equation $f=0$ and let $\left(C_{i}, 0\right), i=1, \ldots, r$, be its reduced branches defined by $f_{i}=0$.

The local curve $C$ defines a link with multiplicities $L:=C \cap S_{\varepsilon}^{3}$, in the sphere of radius $\varepsilon>0$ around $0 \in \mathbb{C}^{2}$, which does not depend on $\varepsilon$ provided $\varepsilon$ is small enough. The link $L$ consists of the components $C_{i} \cap S_{\varepsilon}^{3}$, with multiplicities $q_{i}$ and determines the topological type of the germ $C$. Moreover, Milnor proved that the map $\frac{f}{|f|}: S_{\varepsilon}^{3} \backslash L \rightarrow S^{1}$ is a $C^{\infty}$-locally trivial fibration, the Milnor fibration. Any fibre $F$ of this fibration is called the Milnor fibre of $f$ (see [M, Theorem 4.8]). $A^{\prime}$ Campo $[\mathrm{A}]$ and Eisenbud-Neumann [EN], using different methods, calculated many topological invariants of the fibration $\frac{f}{|f|}$ from the resolution graph or the splicing diagrams. We are only interested in the Euler characteristic $\chi(F)$ of the surface $F$. If $f$ is reduced, i.e. every power $q_{i}$ is equal to one, the Euler characteristic of $F$ is $1-\mu(C, 0)$, where $\mu(C, 0)$ is the Milnor number of the isolated singularity of $C$. Moreover the Euler characteristic of $F$ is related to topological and geometric invariants of its branches by the well-known formula:

$$
\chi(F)=-2 \sum_{1 \leq i<j \leq r}\left(C_{i}, C_{j}\right)_{0}+\sum_{i=1}^{r}\left(1-\mu\left(C_{i}\right)\right),
$$

where $\left(C_{i}, C_{j}\right)_{0}$ is the intersection multiplicity of $C_{i}$ and $C_{j}$ at the origin and $\mu\left(C_{i}\right)$ is the Milnor number of $C_{i}$ at the origin (e.g. see [BK]).

Received by the editors July 24, 1996 and, in revised form, June 27, 1997.

1991 Mathematics Subject Classification. Primary 32S05, 14H20; Secondary 14B05.

Key words and phrases. Euler characteristic, Milnor fibration, Milnor fibre.

This work was done under the partial support of CAYCIT PB94-291. 
On the other hand, when $f$ is non-reduced Schrauwen [S] expressed the Euler characteristic of $F$ in terms of special points of suitable deformations of $f$. For calculating $\chi(F)$ in this case one can use the methods of A'Campo or EisenbudNeumann and construct the resolution graph or the splicing diagram.

The aim of this note is to give a closed formula for the Euler characteristic of $F$ without the construction of these graphs.

For every $q \in \mathbb{N}^{r}$ set

$$
F^{q}:=\left\{z \in S_{\epsilon}: \prod_{1 \leq i \leq r, q_{i} \neq 0}\left(\frac{f_{i}}{\left|f_{i}\right|}\right)^{q_{i}}(z)=1 \text { and } f_{i}(z) \neq 0 \quad \forall i=1, \ldots, r\right\} .
$$

Note that, for $\epsilon$ small, the surface $F^{q}$ is the Milnor fibre of the local curve $C^{q}:=\left\{f_{1}^{q_{1}} \cdots f_{r}^{q_{r}}=0\right\}$ if and only if all $q_{i} \neq 0$. If some $q_{i}$ are zero, but $q \neq 0$, then $F^{q}$ is the Milnor fibre of $\prod_{1 \leq i \leq r, q_{i} \neq 0} f_{i}^{q_{i}}$ with punctures, where the number of punctures equals $\sum_{1 \leq i, j \leq r, q_{i} \neq 0, q_{j}=0}\left(C_{i}, C_{j}\right)_{0}\left(q_{i}\right)$. For $q=0$ the space $F^{q}$ is just the complement of the link of the curve $C$.

Our generalized and closed formula is:

$$
\chi\left(F^{q}\right)=-\sum_{1 \leq i<j \leq r}\left(C_{i}, C_{j}\right)_{0}\left(q_{i}+q_{j}\right)+\sum_{i=1}^{r} q_{i}\left(1-\mu\left(C_{i}\right)\right) .
$$

I am indebted to the referee for suggesting how to improve the presentation of the proof of the formula.

\section{Proof OF THE FORMUlA}

The formula follows from the two following lemmas.

Lemma 1. The function $q \in \mathbb{N}^{r} \rightarrow \chi\left(F^{q}\right)$ is additive.

Proof. Let $\pi: X \rightarrow \mathbb{C}^{2}$ be a proper modification of $\mathbb{C}^{2}$ above the origin such that, for every point on the divisor $E:=\pi^{-1}(0)$, the total transform of the $\bigcup_{1 \leq i \leq r} C_{i}$ has normal crossing singularities. Let $\widetilde{C_{i}}$ be the strict transform of $C_{i}$ by $\pi$ and $E_{\alpha}, \alpha \in A$, the components of $E$.

First assume $q \neq 0$. Put $f^{q}=\prod_{1 \leq i \leq r, q_{i} \neq 0} f_{i}^{q_{i}}$. Observe that $F^{q}$ retracts on $E \backslash\left(\bigcup_{1 \leq j \leq r, q_{j}=0} \widetilde{C_{j}}\right)$. With the formula of A'Campo we get:

$$
\chi\left(F^{q}\right)=\sum_{\alpha \in A} m\left(f^{q}, E_{\alpha}\right) \chi\left(\check{E}_{\alpha}\right),
$$

where $\check{E}_{\alpha}:=E_{\alpha} \backslash\left(\bigcup_{\beta \neq \alpha} E_{\beta} \cup \bigcup_{1 \leq i \leq r} \widetilde{C}_{i}\right)$. Then

$$
\chi\left(F^{q}\right)=\sum_{\alpha \in A} \sum_{i=1}^{r} q_{i} m\left(f_{i}, E_{\alpha}\right) \chi\left(\check{E}_{\alpha}\right),
$$

since $m\left(f^{q}, E_{\alpha}\right)=\sum_{1 \leq i \leq r} q_{i} m\left(f_{i}, E_{\alpha}\right)$.

To prove the additivity it remains to observe that $\chi\left(F^{0}\right)=0$.

Put $e_{i}=(0, \ldots, 1, \ldots, 0)$. From the additivity we get:

$$
\chi\left(F^{q}\right)=\sum_{i=1}^{r} q_{i} \chi\left(F^{e_{i}}\right) .
$$


Lemma 2.

$$
\chi\left(F^{e_{i}}\right)=-\sum_{\substack{j=1, \ldots, r \\ i \neq j}}\left(C_{i}, C_{j}\right)_{0}+\left(1-\mu\left(C_{i}\right)\right) .
$$

Proof. Remember that $F^{e_{i}}$ is the Milnor fibre $F_{i}$ with $\sum_{1 \leq j \leq r, j \neq i}\left(C_{i}, C_{j}\right)_{0}$ punctures.

Remark that Lemma 1 holds for the case where the germs of the curves $C_{i}$ are reduced and have no branch in common. Thus, if we assume

1. each $f_{i}$ has no multiple components (i.e. $f_{i}$ is squarefree) and

2. for $i, j \in\{1, \ldots, r\}, i \neq j$, the germ $f_{i} f_{j}$ has no multiple components, then we finally get for the Euler characteristic of the Milnor fibre of $F$ of $f=$ $f_{1}^{q_{1}} \cdots f_{s}^{q_{s}}, q_{i}>0$, the formula:

$$
\chi(F)=-\sum_{1 \leq i<j \leq s}\left(C_{i}, C_{j}\right)_{0}\left(q_{i}+q_{j}\right)+\sum_{i=1}^{s} q_{i}\left(1-\mu\left(C_{i}, 0\right)\right) .
$$

To have this formula for squarefree factorization is particularly useful for inductive calculations. If $R$ is a computable ring with $\operatorname{char}(R)=0$ and $f$ is a polynomial in $R[x, y]$, then there exists an algorithm that computes a squarefree decomposition of $f$ in $R[x, y]$ (see [BWK, Proposition 2.86, Corollary 2.92]). This is also a squarefree decomposition in $R\{x, y\}$ and one may then compute the intersection multiplicities and the Milnor numbers. I would like to thank Bernd Martin for showing me the implementation of this algorithm using the computer algebra system SINGULAR, [GPS].

\section{REFERENCES}

[A] N. A'Campo, La fonction zeta d'une monodromie, Comm. Math. Helvetici 50 (1975), 233-248. MR 51:8106

[BWK] T. Becker, V. Weispfenning and H. Kredel, Gröbner Bases: A Computational Approach to Commutative Algebra, Graduate Text in Math., vol. 141, Springer-Verlag, BerlinHeidelberg-New York, 1991. MR 95e:13018

[BK] E. Brieskorn and H. Knörrer, Plane algebraic curves, Birkhäuser, Basel-Boston-Stuttgart, 1986. MR 88a:14001

[EN] D. Eisenbud and W. Neumann, Three-dimensional link theory and invariants of plane curve singularities, Ann. of Math. Studies, No. 110, Princeton Univ. Press, Princeton, N.J., 1985. MR 87g:57007

[GPS] G.M. Greuel, G. Pfister, H. Schoenemann, SINGULAR. A computer algebra system for singularity theory and algebraic geometry, It is available via anonymous ftp from helios.mathematik.uni-kl.de.

[M] J. Milnor, Singular points of complex hypersurfaces, Ann. of Math. Studies, No. 61, Princeton Univ. Press, Princeton, N.J., 1968. MR 39:969

[S] R. Schrauwen, Deformations and the Milnor number of non isolated plane curve singularities, Singularity theory and its applications. Part I, proceedings Warwick 1989. Lecture Notes in Math., vol. 1462, Springer-Verlag, Berlin-Heidelberg-New York, 1991, pp. 276291. MR 92j:32128

Departamento de Algebra, Facultad de CC. Matemáticas, Universidad Complutense DE MADRID, MADRID 28040, SpaIN

E-mail address: amelle@eucmos.sim.ucm.es 